\title{
The Study of Routing Protocol for UAV Flock Network
}

\author{
Wenjing Yang ${ }^{a}$, Haiyan Zhaob \\ College of Information and Communications, National University of Defense Technology, Xi'an, Shaanxi, \\ 710106, China \\ a13086903@qq.com, b23414388@qq.com
}

Keywords: Unmanned aerial vehicle, networking technology, routing protocol

Abstract: This paper studies the networking technology and routing protocol which is commonly used in UAV ad hoc networks, provides profound understanding about the working principle of the routing algorithm and proposes a routing algorithm based on minimal ID clustering. This method can reduce the cost of routing algorithm and flooding broadcast, which is suitable for high dynamic UAV fleet networking.

\section{Introduction}

UAVs are widely used in military fields around the world today, including reconnaissance, cruising and rapid combat. Based on such factors as strike radius, attack accuracy, information sharing and others, UAV formation network has gained more and more popularity. Many countries, especially all the major military countries in the world, vied for it as the focus of weapon development.

UAV network is a dynamically changing wireless mobile communication system, so the system is faced with complicated situations. As designed in a wireless high-speed mobile environment, it is most important for a UAV network system to select the appropriate networking method and appropriate routing technology. Although the international research on MANET technology has been carried out for many years, the nodes usually have slow moving speed, which is approximately several meters to ten meters per second. However, the moving speed of the drone is generally about tens of meters per second. In other words, UAV and terrestrial mobile terminals vary greatly in speed, resulting in UAV network topology changes too frequently, thus seriously affecting the performance of the network. Therefore, it is necessary to put forward higher requirements on UAV network routing strategy and carry out relevant research specially.

\section{OLSR routing protocol}

OLSR routing protocol is a commonly used protocol of UAV, of which the core idea is multi-point relay mechanism. The number of broadcast packets in Ad Hoc network has been sufficiently controlled by selective flooding. This approach greatly reduces the number of TC packets being forwarded.

OLSR routing protocol establishes local link information base and the adjacent area information base through the periodic HELLO packet interaction. The HELLO packet format is shown in Figure 1 .

\begin{tabular}{|c|c|c|}
\hline \multicolumn{2}{|c|}{8} & 16 \\
\hline Reserved & Htime & Willingness \\
\hline Link Code & Time & Link Message Size \\
\hline \multicolumn{3}{|c|}{ Neighbor Interface Address } \\
\hline \multicolumn{3}{|c|}{ Neighbor Interface Address } \\
\hline \multirow{3}{*}{ Neighbor Interface Address ..... }
\end{tabular}

Figure 1 The HELLO format 
The TC format is shown in Figure 2.

\begin{tabular}{|c|}
\hline \multicolumn{2}{|c|}{16} \\
\hline ANSN \\
\hline Advertised Neighbor Main Address \\
\hline Advertised Neighbor Main Address \\
\hline$\ldots \ldots \ldots$ \\
\hline$\ldots \ldots \ldots$ \\
\hline
\end{tabular}

Figure 2 The TC format

OLSR Routing Protocol Table

- Local Link Table

The local link table holds the link information between the node and its neighbors. The table is saved as shown in Figure 3 below.

\begin{tabular}{|l|l|l|l|l|}
\hline L_local_iface_addr & L_neighbor_iface_addr & L_SYM_Time & L_ASYM_Time & L_Time \\
\hline
\end{tabular}

Figure 3 The local link table holds the link information

- The Neighborhood Information

Neighbor information table contains neighbor information, two-hop neighbor information, MPR information and MPR selector information

(1) The specific storage format of the neighbor table of the node is shown in Figure 4.

$$
\begin{array}{|l|l|l|}
\text { N_neighbor_main_addr } & \text { N_Status } & \text { N_willingness }
\end{array}
$$

Figure 4 The storage format of neighborhood table

(2) The specific storage format of two-hop neighbor table is shown in Figure 5.

\begin{tabular}{|l|l|l|}
\hline N_neighbor_main_addr & N_2hop_addr & N_time \\
\hline
\end{tabular}

Figure 5 The storage format of two-hop neighbor table

This paper studies the commonly used OLSR routing protocol and provides in-depth understanding of the routing algorithm works.

- MPR Selector information table

The storage format of how MPR selecting node information table is shown in Figure 6 below.

$$
\begin{array}{|l|l|}
\hline \text { M_main_addr } & \text { MS_time } \\
\hline
\end{array}
$$

Figure 6 the storage format of how MPR selecting node information table

However, the traditional OLSR routing protocol cannot be used when the UAV does not maintain formation of high-dynamic network communications. Especially when the network is large, the use of clustering network structure, can reduce the routing algorithms and flooding broadcast, conveniently manage mobile nodes, control nodes access to wireless channels, and can improve network scalability and Qos security capabilities. The choice of clustering algorithm depends on the needs of the application, the environment of the network, and the characteristics of the nodes. Different clustering algorithms have different optimization objectives, including minimizing cluster calculation and maintenance, minimizing cluster heads, maximizing cluster stability, and maximizing node lifetime. CBRP uses the smallest ID algorithm for clustering.

The minimum ID algorithm is a simple clustering algorithm proposed by Greta and Tsai. Each node allocates a unique ID throughout the network, periodically broadcasts Hello messages including its ID value to other neighboring nodes, during which the node with the smallest ID among the adjacent nodes serves as the cluster head. This kind of clustering algorithm has features like simple design, small amount of calculation, easy implementation and fast convergence. The 
minimum ID algorithm has a slower updating of cluster heads, less overhead of maintaining clusters, and a higher throughput of the network due to the more reasonable number of nodes in clusters and clusters. The disadvantage of this algorithm is that it tends to select nodes with smaller IDs as cluster heads, which will consume more energy for these nodes and reduce the node lifetime when the energy is limited, and the algorithm does not consider such factors as load balancing.

\section{Other technologies for drone networks}

\subsection{MAC scheduling method}

MAC design is one of the key technologies of wireless network. TDMA is an access control method that divides each member's sending time by time slice. It divides the time axis into moving length, repeated time frames, eliminating collisions and reducing energy consumption of nodes. In this paper, a distributed TDMA protocol is used as the MAC scheduling method for UAV networks.

The corresponding scheduling methods will change according to different modes of UAV network. As Unicom's peer-to-peer model is simple in structure, all nodes are peer-to-peer structures and adopt the same MAC. Therefore, each node needs to be assigned a corresponding time slot by using the traditional TDMA method. For clustered hierarchical mode, MAC scheduling is divided into two parts: cluster scheduling and intra-cluster scheduling, as shown in the figure.

\subsection{Relay transmission technology}

When beyond the radio coverage of the ground control station, UAVs will use relay to achieve communication with each other and with the ground control station. In accordance with the location of the relay device, relay can be divided into ground relay and air relay. Terrestrial repeater equipment is placed on the ground control station and drone between the commanding heights, air trunks are used as UAV repeater or satellite relay forwarding. The air relay cost higher than the relay mode. The repeater mode takes $U A V$ as a relay device, and uses the ground station, relay UAVs, mission UAVs to form a network of over-the-horizon communication, as shown in Figure 1.2. It is characterized by fast moving speed, high maneuverability, low space limitations and low cost for radio waves, but the need to enhance the survivability of relay UAVs. Compared with the UAV relay mode, the satellite relay covers a wider area, and the satellite channel has relatively stable performance, available frequency bandwidth and larger communication capacity, but the cost is comparatively higher.

\subsection{Communication antenna}

The omnidirectional antenna is simple in structure and can easily meet the high-speed moving characteristics of the UAV node, and it also meets the complicated requirements of the UAV application environment, ensure the stability of the antenna performance and the design of the tracking device is relatively simple.

Directional antennas have high-performance, low-sidelobe pattern characteristics, with the advantages of high spatial multiplexing, long transmission distances, high transmission rates, low intercept and low detection.

UAV data link should be a combination of omnidirectional antenna and directional antenna, in order to give full play to their advantages, increase the physical layer performance, which can further improve the UAV network communication performance.

Antenna alignment means that the main lobe beams of the two antennas cover each other to achieve the maximum antenna gain. Antenna tracking uses antenna tracking devices and algorithms. While the positions of the two antennas are relatively changed, the antennas are always aligned with each other to reduce External interference and room interference.

In the initial stage of network initialization, omnidirectional antenna search ought to be used to find the location of neighboring nodes and network topology information due to the incomplete topology information and other reasons. The directional antenna obtains the maximum antenna gain by performing fast scanning in a small area to achieve the antenna alignment on the basis of 
obtaining the node location information of the omnidirectional antenna. Information like pitch angle, swing angle, attitude correction and other parameters should be included in the antenna alignment and tracking algorithm in order to get better performance, and ensure the communication dual hair antenna alignment.

\section{References}

[1] L. X. Hu, D. Evans. Using directional antennas to Prevent wormhole attacks[C]. Network and Distributed System Security Symposium Proceedings, San Diego, 2004, 1-11

[2] K. Sanzgiri, B. Dahill, B. N. Levine, et al. A Secure Routing Protocol for Ad hoc Network[C]. 10th IEEE International Conference on Network Protocols Proceedings, Paris, 2002, 78-87

[3] P. Jacquet, P. Muhlethaler, T. Clausen, et al. Optimized link state routing protocol for ad hoc networks[C]. Multi Topic Conference Proceedings, Lahore, 2001, 62-68

[4] V. D. Park, M. S. Corson. Highly Adaptive Distributed Routing Algorithm for Mobile Wireless Networks[C], 16th IEEE INFOCOM Proceedings, Kobe, 1997, 1405-1414

[5] A. S. Alshahrani. Rushing attack in mobile ad hoc networks[C]. Third International Conference on Intelligent Networking and Collaborative Systems, Fukuoka, 2011, 752-758 